\title{
MBMW-201: The next generation multi-beam mask writer (Conference Presentation) (Withdrawal Notice)
}

Christof Klein, Hans Loeschner, Elmar Platzgummer

Christof Klein, Hans Loeschner, Elmar Platzgummer, "MBMW-201: The next generation multi-beam mask writer (Conference Presentation) (Withdrawal Notice)," Proc. SPIE 10958, Novel Patterning Technologies for Semiconductors, MEMS/NEMS, and MOEMS 2019, 109580K (2 December 2019); doi: 10.1117/12.2516033

SPIE. Event: SPIE Advanced Lithography, 2019, San Jose, California, United States 


\section{MBMW-201: The next generation multi-beam mask writer (Conference Presentation) (Withdrawal Notice)}

Christof Klein, Hans Loeschner, Elmar Platzgummer

Medizinische Univ. Wien (Austria)

Proc. SPIE 10958, 109580K (2019)

Online Publication Date: 16 August 2019

Withdrawn from Publication: 2 December 2019

Conference Date: 25 February-28 February 2019

Conference Location: San Jose, California, United States

Conference Title: Novel Patterning Technologies for Semiconductors, MEMS/NEMS and MOEMS 2019

Conference Chairs: Martha I. Sanchez

Publisher's Note: This video, originally published on 16 August 2019, was withdrawn per author request. 